\title{
Transfusion-related acute lung injury: A dangerous and underdiagnosed noncardiogenic pulmonary edema
}

\author{
Krzysztof Jaworski ${ }^{1}$, Krystyna Maślanka ${ }^{2}$, Dariusz A. Kosior ${ }^{1,3}$ \\ ${ }^{1}$ Department of Noninvasive Cardiology and Hypertension, \\ Central Clinical Hospital of the Ministry of Interior, Warsaw, Poland \\ ${ }^{2}$ Department of Immunohaematology and Immunology of Transfusion Medicine, \\ Institute of Hematology and Transfusion Medicine, Warsaw, Poland \\ ${ }^{3}$ Faculty of Health Sciences, University of Radom, Poland
}

\begin{abstract}
Transfusion-related acute lung injury (TRALI) is one of the leading causes of death associated with transfusion of blood and blood components. The understanding of the etiology and pathophysiology of this syndrome has much improved during the last decades, nevertheless numerous issues are still unresolved and symptomatic treatment remains the cornerstone of medical management. Consequently more attention is directed at primary as well as secondary prevention. The awareness of the problem within the medical society is still unsatisfactory which results in a high number of unrecognized cases or of inaccurate diagnoses one of which is cardiogenic pulmonary edema. The aim of this review is to make the TRALI syndrome more familiar to clinicians and to emphasize how significant proper medical management is both for the patients presenting TRALI symptoms as well as for future recipients of blood components. (Cardiol J 2013; 20, 4: 337-344)
\end{abstract}

Key words: transfusion, pulmonary edema, leukocyte antibodies, respiratory distress

\section{Introduction}

Since the first successful blood transfusion performed by James Blundell in 1818, this procedure has become part of daily clinical practice. Advances in blood safety have greatly reduced the risk of transfusion-associated infectious disease transmission and blood has never been as safe as it is now, however, the risks associated with blood transfusion have not been entirely eliminated. One of them is the syndrome of acute lung injury (ALI) which leads to respiratory distress. This complication is the subject of our review. The first case of noncardiogenic pulmonary edema following transfusion was described by Bernard in 1951 [1]. Thirty years later, Popovsky and Moore [2] suggested to give this complication the name of TRALI - transfusion-related acute lung injury. During the conference in Toronto in 2004, a panel of experts from the National Institute of Health finally established a clinical definition of TRALI based on ALI temporally associated with transfusion and characterized by dyspnea, hypoxemia and bilateral infiltrates on a chest X-ray [3]. This life-threatening reaction may be caused by any blood component that contains plasma $[4,5]$. TRALI was

Address for correspondence: Krzysztof Jaworski, MD, Department of Noninvasive Cardiology and Hypertension,

Central Clinical Hospital of the Ministry of Interior, ul. Wołoska 137, 02-507 Warsaw, Poland,

tel/fax:+48 2250816 70/+48 2250816 80, e-mail: krjaworski@wp.pl

Received: 18.12 .2012

Accepted: 19.03.2013 
most frequently observed following transfusions of whole blood, red blood cell concentrates, fresh frozen plasma and platelet concentrates $[5,6]$. In some reports the volume of plasma sufficient to induce TRALI is estimated at $10-15 \mathrm{~mL}[7,8]$. The complication affects all age groups and occurs with the same frequency in both men and women [4]. According to the Food and Drug Administration statistics TRALI is responsible for $43 \%$ of transfusion-related deaths reported in 2007-2011 and it is the leading cause of mortality associated with this procedure $[9,10]$. TRALI risk is estimated at approximately $0.02 \%$ per unit of blood component or $0.16 \%$ per recipient $[4,11,12]$. Shaz et al. [13] estimate the incidence of this syndrome at $1 / 260000$ transfusions. The true TRALI prevalence rate, however, remains unknown due to a large number of cases unreported to blood transfusion centers. Much too often, dyspnea and hypoxia observed during transfusion are attributed to volume overload or cardiogenic pulmonary edema while mild cases are entirely disregarded. This precludes the opportunity for confirmation of TRALI in tests as well as implementation of appropriate preventive measures.

\section{Etiology and pathophysiology}

Pathophysiology of TRALI is not fully understood. Two mechanisms which lead to the development of the syndrome are distinguished as reflected in the following classification:

- immune-mediated TRALI, predominantly caused by anti-HLA (human leukocyte antigen) antibodies class I, II and/or less frequent antibodies directed against specific antigens of granulocytes - HNA (human neutrophil antigen), present in the serum of the recipient or donor, which react with the donor's or recipient's leukocytes respectively $[4,5,7,12-15]$.

- non-immune-mediated TRALI, which may be attributed to transfusion of biologically active compounds accumulated in stored blood components such as bioactive lipids, proinflammatory cytokines or platelet microparticles with high procoagulant activity. A high potassium level is considered an additional risk factor [4, 13-20].

Leukocyte antibodies are currently believed to be crucial for TRALI development. The cognate antigens for these antibodies are human leukocyte antigens (either HLA or HNA) expressed on the recipient's/donor's own blood cells. The main cells involved in TRALI pathogenesis are neutrophils.
The interaction between the antibody and its cognate antigen is thought to induce neutrophil activation, sequestration and finally, endothelial barrier damage. Several interesting TRALI cases have been reported where antibodies of one donor activated the leukocytes of another donor in the bloodstream of the recipient [21, 22]. Dykes et al. [23] described a case of TRALI in a patient with a single lung transplant. Following transfusion of red blood cell concentrate the TRALI syndrome developed only in the transplanted organ, because contrary to other tissues, it contained the HLA-B44 antigen reactive to donor's antibodies. This is strong evidence for immunological etiology of TRALI. Leukocyte antibodies differ as to their specificity, degree of activity, as well as ability to induce agglutination of leukocytes. For example, antibodies to HNA-3a and HLA-A2 antigens which have proagglutination properties correlate with increased morbidity and mortality, whereas no TRALI related deaths were reported due to antibodies directed against HNA-1a and HNA-2a [16].

Immune-mediated TRALI, where antibodies have been detected in at least one of the transfused blood components, are estimated at $65-85 \%$ of all reported TRALI cases [4,5]. Cases where no antibodies were detected despite the manifestation of characteristic clinical symptoms are considered "non-immune-mediated" TRALI. It should also be noted that TRALI occurs only in some recipients of blood components that contain antibodies. It is still unclear why leukocyte antibodies cause this complication in some patients while not in others. This is usually associated with lack of cognate leukocyte antigens in recipient's/donor's blood. These observations were confirmed by the "look back" procedure described by Maślanka et al. [24] where no TRALI reaction was reported for 26 patients transfused with blood containing HLA antibodies although cognate leukocyte antigens were detected in the blood of 11/26 recipients. Previously, the same antibodies had caused a TRALI reaction in another patient. Perhaps the crucial issue here is the antibody titer, affinity, avidity or density of cognate antigenic epitope on leukocyte cells.

However, clinical evidence indicates that most patients are critically ill prior to TRALI onset. There are additional risk factors such as mechanical ventilation, sepsis, shock, hematologic malignancy, surgery (e.g. cardiac, orthopedic), alcohol abuse and active smoking [8, 10-12]. In light of these observations Silliman et al. [25] postulated a hypothesis of a "two-hit" model. This model assumes that TRALI onset depends on the coexistence 
of a factor that predisposes a recipient's reaction as well as on the presence of leukocyte-activating compounds in transfused blood. The "first hit" is provoked by proinflammatory condition, e.g. pneumonia, sepsis, which induces pulmonary endothelial activation that promotes neutrophil sequestration and adhesion to pulmonary capillary walls. These alterations in the lung vessels are insufficient to induce TRALI but they prepare the ground for TRALI. The "second hit" is provided by transfusion of a blood component that contains either leukocyte antibodies or biologically active lipids and cytokines. These provoke neutrophil agglutination or induce reactive neutrophils to secrete proinflammatory mediators such as cytokines (IL-1, IL-6, IL-8, TNF-alpha), release proteolytic enzymes (e.g. elastase, azurocidin), and produce reactive oxygen species [4, 16, 25]. This initiates the cascade of immune reactions with subsequent damage to the vascular endothelium and pulmonary capillary leakage of proteinaceous fluid in the alveolar space [5, 13-15]. Additionally, Silliman et al. [17] demonstrate that in the "two hit" model leukocytes may be directly stimulated by biologically active substances accumulated in stored blood components which include: arachidonic acid, lyso-phosphatydylocholine (LysoPCs) and a soluble form of CD40 ligand ( $\mathrm{SCD} 40 \mathrm{~L})$ [5, 13, 14]. Silliman's observations are confirmed by Covin et al. [26] who described an onset of TRALI in a patient after an autologous transfusion where no antibodies were detected in blood but leukocyte activation was induced by accumulation of active lipids. However, recent clinical observations and $e x$ vivo studies question the involvement of biologically active lipids and SCD40L in TRALI development [27-29].

According to Bux and Sachs [14], the authors of the threshold model for TRALI, which was presented in 2007 , in the "two hit" mechanism the reaction occurs only if activation of neutrophils exceeds a critical stage. In patients with multiple risk factors this reaction is triggered at lower antibody titre than in patients with no risk factors, as each risk factor contributes to the reduction of the threshold of susceptibility to TRALI. The above hypotheses are not necessarily mutually exclusive but may coexist and present a similar clinical picture.

\section{Clinical presentation}

TRALI is characterized by acute respiratory failure observed during transfusion or within $6 \mathrm{~h}$ of transfusion. The main symptom is dyspnea, which often co-occurs with tachypnea, tachycardia, cyanosis and frothy pulmonary secretions. Fever, hypotension or hypertension are also reported [6, $25,30]$. Decreased breath sound and diffuse crackles over the lung fields are revealed in physical examination [25]. Respiratory failure is corfirmed by hypoxemia (usually $30-50 \mathrm{~mm} \mathrm{Hg}$ ) detected in arterial blood gas. As these are nonspecific symptoms, TRALI recognition is based on exclusion of other causes such as anaphylactic reaction, cardiogenic pulmonary edema, acute respiratory distress syndrome (ARDS) or bacterial infection.

Basic laboratory tests are not considered diagnostic criteria, because of their low sensitivity and specificity. Occasionally leukopenia, neutropenia, hypoalbuminemia and hypocomplementemia can be detected $[3,4,6]$. Although fairly typical, leukopenia associated with leukocyte sequestration in the pulmonary circulation is usually temporary and often passes unnoticed [8]. Recent reports emphasize the diagnostic significance of brain natriuretic peptide (BNP) and it is recommended to determine the BNP concentration in each and every case of post-transfusion respiratory failure [27, 31].

TRALI frontal chest radiograph manifests bilateral fluffy infiltrations with no cardiomegaly. Hemodynamic monitoring helps to differentiate TRALI and cardiogenic pulmonary edema. Normal or low pulmonary capillary wedge pressure and central venous pressure values are characteristic for TRALI $[4,30]$.

\section{Diagnosis}

One should always suspect TRALI if dyspnea and hypoxia occur within a short time of transfusion of any blood component. During the American-European Consensus Conference in 2004 a group of experts established diagnostic criteria for TRALI (Table 1) [3].

A full diagnostic procedure requires that each TRALI case is reported to a reference center which performs serological testing for antibodies directed against HLA class I, class II, and HNA in recipient's and donor's blood. The Working Party on Granulocyte Immunobiology of the International Society of Blood Transfusion recommends the lymphocytotoxicity test (LCT) for HLA antibody detection and one of the following tests for HLA non-complement binding antibodies: enzyme assays (ELISA - enzyme-linked immunosorbent assay), immunofluorescence test or luminex technique. A combination of granulocyte immunofluorescence test (GIFT) and granulocyte agglutination 
Table 1. Diagnostic criteria for transfusion-related acute lung injury (TRALI) [3, in our modification].

\begin{tabular}{|c|c|}
\hline TRALI & Possible TRALI \\
\hline 1. Acute lung injury: & 1. Acute lung injury: \\
\hline A. Acute onset of symptoms & A. Acute onset of symptoms \\
\hline $\begin{array}{l}\text { B. Hypoxemia: } \mathrm{PaO}_{2} / \mathrm{FiO}_{2}<300 \text { or } \mathrm{SpO}_{2}<90 \% \\
\text { (no oxygen) or other clinical signs of hypoxemia }\end{array}$ & $\begin{array}{l}\text { B. Hypoxemia: } \mathrm{PaO}_{2} / \mathrm{FiO}_{2}<300 \text { or } \mathrm{SpO}_{2}<90 \% \\
\text { (no oxygen) or other clinical signs of hypoxemia }\end{array}$ \\
\hline C. Bilateral infiltrates on frontal chest radiograph & C. Bilateral infiltrates on frontal chest radiograph \\
\hline D. No evidence of left atrial hypertension & \\
\hline 2. No preexisting acute lung injury before transfusion & 2. No preexisting acute lung injury before transfusion \\
\hline $\begin{array}{l}\text { 3. Development of symptoms during or within } \\
6 \mathrm{~h} \text { of transfusion }\end{array}$ & $\begin{array}{l}\text { 3. Development of symptoms during or within } \\
6 \mathrm{~h} \text { of transfusion }\end{array}$ \\
\hline $\begin{array}{l}\text { 4. No temporal association with alternative risk } \\
\text { factors for acute lung injury (foreign body } \\
\text { aspiration, pneumonia, inhalation of toxins, } \\
\text { burns, drowning, polytrauma, drug overdose, } \\
\text { acute pancreatitis, cardiopulmonary bypass) }\end{array}$ & $\begin{array}{l}\text { 4. Possible temporal association with alternative } \\
\text { risk factors for acute lung injury (foreign body } \\
\text { aspiration, pneumonia, inhalation of toxins, } \\
\text { burns, drowning, polytrauma, drug overdose, } \\
\text { acute pancreatitis, cardiopulmonary bypass) }\end{array}$ \\
\hline
\end{tabular}

test (GAT) is usually used for anti-HNA antibody detection while the positive results are verified by monoclonal antibody immobilization of granulocyte antigens (MAIGA) [31, 32]. When the presence of antibodies in the serum of recipient/donor is confirmed, the subsequent step involves determination of the corresponding antigen in the donor/recipient or a cross-match between recipient's (or donor's) leukocytes and donor's (or recipient's) plasma. A positive result confirms the diagnosis of immune-mediated TRALI [8, 31]. For research purposes the capacity of antibodies to activate leukocytes is sometimes measured in tests of neutrophil respiratory burst following incubation of plasma suspected of inducing TRALI with neutrophils of healthy people [3].

It should be emphasized that antibody detection is not indispensable for TRALI diagnosis which is primarily based on the clinical picture [3, 27, 33]. The diagnostic algorithm is presented in Figure 1.

\section{Differential diagnosis}

The criteria for TRALI diagnosis presuppose the exclusion of all other causes leading to acute respiratory distress syndrome, such as: cardiogenic pulmonary edema, pneumonia, bacterial contamination of blood components or acute hemolytic reaction.

The major difficulty remains to differentiate TRALI from transfusion-associated circulatory overload (TACO), which is a result of excessive volume of transfused fluid. The clinical picture of TACO resembles that of cardiogenic pulmonary edema. Apart from dyspnea and tachypnea, the characteristic features are jugular venous distension,

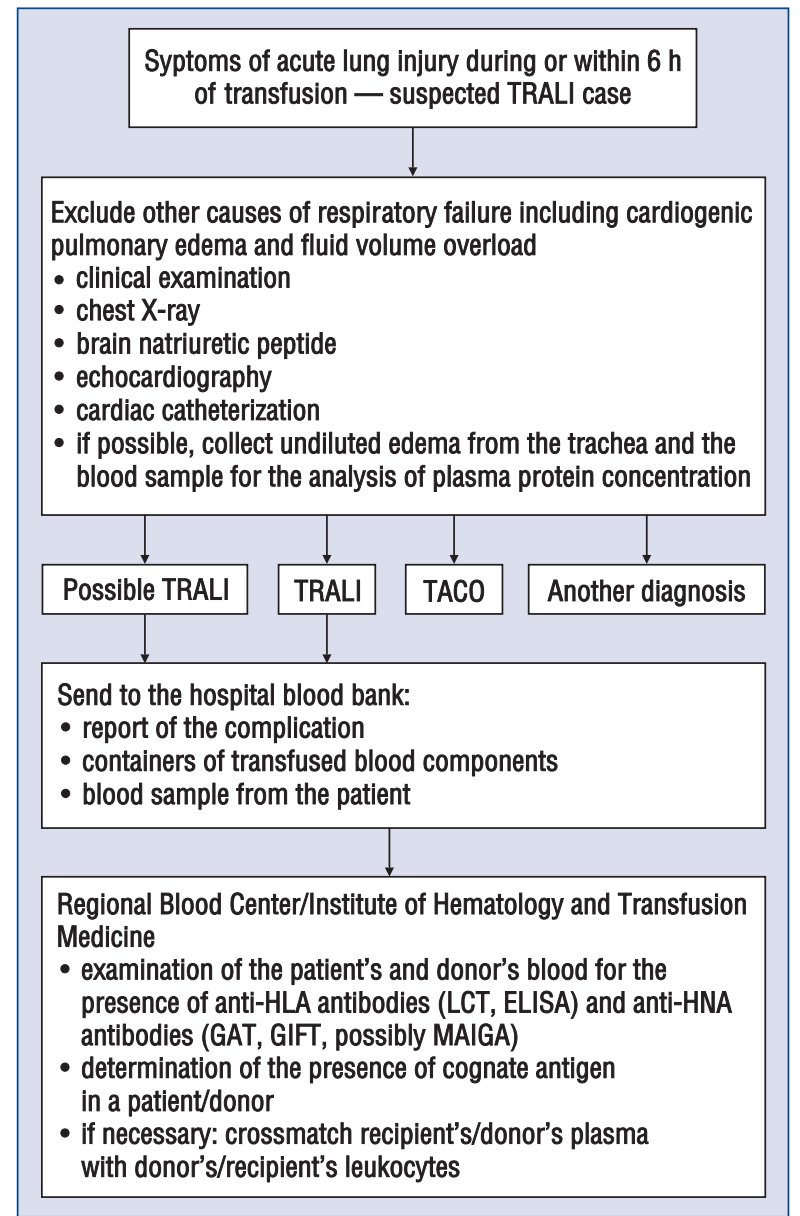

Figure 1. Transfusion-related acute lung injury (TRALI) diagnostic algorithm; TACO - transfusion-associated circulatory overload; HLA - human leukocyte antigen; HNA - human neutrophil antigen; LCT — lymphocytotoxicity test; ELISA - enzyme-linked immunosorbent assay; GAT — granulocyte agglutination test, GIFT — granulocyte immunofluorescence test; MAIGA — monoclonal antibody immobilization of granulocyte antigens. 
S3 gallop and high systolic blood pressure [3]. Gajic et al. [34] proposed an algorithm which is useful in distinguishing between these 2 conditions. It is based on measurement of BNP, the ratio of protein concentration in the bronchial fluid and in the plasma as well as the assessment of myocardial function. TACO as the cause of respiratory failure can be excluded with a high degree of probability when the BNP value is $<250 \mathrm{pg} / \mathrm{mL}$ or its posttransfusion-to-pretransfusion ratio $<1.5$. Index protein in edema fluid/protein in serum concentration $<0.65$ supports the hydrostatic pulmonary edema, while the rate of $>0.75$ indicates excessive endothelial barrier permeability. Fluid should be collected immediately after intubation, preferably within $15 \mathrm{~min}$ of the onset of symptoms, because if later, water is resorbed and the results of tests are false positive due to protein concentration in the alveoli [12]. When circulatory overload occurs the patient's condition can rapidly be improved by diuretic treatment [25]. The estimated mortality rate is between 5-15\% [34]. It should be noted that TACO and TRALI can coexist, moreover there is some evidence that TACO predisposes TRALI development by virtue of pro-inflammatory effect of high pulmonary capillary pressure [35].

\section{Treatment}

When symptoms of acute respiratory distress occur during transfusion, the procedure should immediately be discontinued and not resumed even if the symptoms diminish [36]. Treatment of TRALI is symptomatic and based on oxygen therapy $[4,5]$. Approximately $70 \%$ of patients require intubation and mechanical ventilation [5]. The strategy of low tidal volumes and low plateau pressure used in the treatment of ARDS appears to be proper also for TRALI [8]. Reports of successful use of plasmapheresis and extracorporeal membrane oxygenation are rather scarce $[37,38]$. There are numerous attempts to use glucocorticoids in clinical practice but there is little scientific evidence to support this $[4,38]$. The same can be said for the effect of inhaled nitric oxide - clinical trials seem to deny its potential benefits [39]. Another issue of particular significance for the management of cardiogenic pulmonary edema, is the negative effect of diuretics, which might impair prognosis [4, 38]. The role of other drugs in TRALI management such as synthetic surfactant is still unclear and requires further clinical evaluation [4].

\section{Prognosis}

Although the clinical picture of TRALI is indistinguishable from ALI or ARDS due to other than transfusion causes, the mortality rate for ARDS is $29-70 \%$ while for TRALI it is between $5 \%$ and $10 \%$ [30, 35]. In $80 \%$ of cases the patient's condition improves within 48-96 h while in others the infiltrates in the X-ray persist and the patients require prolonged ventilatory support. Permanent lung injury is very rare $[6,39]$. On the other hand, there is some scientific evidence that in patients in severe condition TRALI is an independent predictor of mortality, a prolonged hospital stay and reduced survival rate. It does not, however, affect the quality of life assessed by the SF-36 Health Survey one year after discharge from the hospital [40].

\section{Prevention}

As there are so few therapeutic options for TRALI, the importance of prevention is growing. Up to date, however, no consensus has been established as to the most effective preventive strategy. Different countries follow different recommendations. One of the commonly accepted methods is deferral from donation of people immunized by leukocyte antigens during previous transfusions or pregnancy $[4,27,32]$. Various sources estimate the probability of immunization during transfusion at about $1-12 \%$ [5]. In a study involving 8,000 blood donors, Kakaiya et al. [41] dispute the idea of donor screening prior to transfusion as they found no statistically significant difference in the incidence of HLA antibodies in patients previously treated or untreated with blood components.

Pregnancy must therefore be considered the main source of immunization. From $4 \%$ to $8 \%$ of women produce HLA antibodies during their first pregnancy, and the probability of immunization increases with each subsequent pregnancy, amounting to about $15 \%$ and $24-26 \%$ after 2 and 3 pregnancies [24, 42]. In 2003, the English National Blood Service adopted the strategy of diverting plasma from female donors for production of fresh frozen plasma which significantly reduced the TRALI incidence rate in the UK (from 36 cases in 2003 to 13 cases in 2009) [43, 44]. Many countries, e.g. France, Germany, Italy, Switzerland, USA, achieved similar results using the strategy for selection of fresh frozen plasma donors, namely the deferral of previously transfused patients and multiparous women or women with leukocyte antibodies [32]. 
There are experts who fear that in some countries this strategy might cause a significant reduction in the number of donations (from $5 \%$ to $30 \%$ ) [3, $14,25,36]$. On the other hand, various studies report that HLA antibodies are present in 1-7\% of population that have never been subjected to alloexposure [5]. This phenomenon has not been explained yet. It is believed that the HLA antibodies in such patients may be autoantibodies or antibodies acquired as result of exposure to bacteria or vaccines antigens $[41,45]$. Moreover, some people may simply be unaware of prior alloexposure (pregnancy ended with miscarriage or childhood transfusion). Prospective studies demonstrate that HLA antibodies rarely lead to the TRALI onset, therefore the deferral of all immunized people would contribute to unneccessary reduction in the number of blood donors which is already below demand [5]. On the other hand, screening of all donors for HLA antibodies would be a time-consuming and costly strategy as well as very difficult to execute.

Unlike HLA antibodies, HNA-3a antibodies are associated with a more severe clinical course. In 2008, the International Society of Blood Transfusion recommended all persons with HNA-3a antibodies to be deferred from blood donation [5]. Undoubtedly the deferral from the further donation should include donors implicated in a TRALI case if leucocyte antibodies were detected in their blood [4, 5, 27]. It is therefore of extreme importance to register all suspicious cases of TRALI. Unfortunately, TRALI cases are still underreported to the blood centers which impedes testing and prevention. The significance of the problem and the proper retrospective procedure that should be applied is described by Kopko et al. [46] in a case report of fatal TRALI associated with fresh frozen plasma transfusion from a 54 -year-old multiparous woman, a multiple blood donor (290 donations in 15 years). Tests performed after the tragic event revealed anti-neutrophil antibodies in her blood. A retrospective inquiry which included 73 donations and 36 recipients of the blood components revealed that 13 of them might have had TRALI [46]. However, not all the cases were reported to the relevant authorities. The outcome of this investigation clearly shows the great responsibility of the transfusion staff and the extent to which the fate of the future recipients relies on the physician's decision to report the TRALI incident.

With the 2-factor etiology in mind, another possibility of TRALI prevention is the modification of "sensitizing" factors, e.g. by applying the strategy of small tidal volumes in mechanically ventilated patients prior to elective transfusion [5].

Many countries (Poland included) try to reduce the incidence rate of posttransfusion immune responses by issuing for clinical use leukoreduced red blood cell concentrates. The process of leukoreduction decreases the number of leukocytes as well as the cytokines which they produce and this may be of significance for recipients with HLA antibodies [5]. Immunized patients therefore should only be given leukoreducedred blood cell or platelet concentrates [27]. Nevertheless, studies by Silliman et al. [17] have demonstrated that leukoreduction does not protect recipients against non-immune-mediated TRALI because the procedure is effective for reduction of accumulated lipid compounds only in a small degree. The source of lipid compounds are platelets in case of LysoPCs and erythrocytes in case of 5-HETE, 12-HETE, 15-HETE as well as arachidonic acid.

There is clinical evidence that TRALI incidence is reduced by using plasma from a pool of 500 to 1,600 donations. Leukocyte antibodies in such blood components are undetectable due to dilution and neutralization by the dissolved HLA antigens $[5,13]$.

There is ongoing research on how to improve the conditions in which blood components are prepared and stored. In a growing number of countries, Poland included, the plasma volume in platelet concentrates is reduced and replaced by additive solutions such as SSP + and PAS IIIM [32]. The Regional Blood Transfusion Center in Warsaw is the only place in Poland where blood components are stored according to a new metod based on the Mirasol PRT system [47]. In the period July 2009-November 2011, approximately 14,500 units of platelet concentrates and 63,000 units of fresh frozen plasma were issued for clinical use and transfused. No TRALI reactions were observed.

TRALI recurrence is accidental or results from higher predisposition of the recipient. While TRALI incidence is estimated at 1:5000 transfusions, the risk of 2 episodes in the same patient with no associated risk factors is statistically 1:25 million [48]. Nevertheless, some authors postulate preventive measures for patients with TRALI history and recommend to use only fresh, leukoreduced, washed red blood cell and platelet concentrates stored for less than 2 days. Such measures will contribute to effective reduction of TRALI risk, regardless of pathogenesis [48, 49].

However, the most important preventive measure for TRALI is the promotion of proper strategy for transfusion of blood components [50]. 
Indications for transfusion should be considered very carefully and it should always be regarded only as a life-saving procedure. Transfusion of large numbers of red blood cell concentrates or fresh frozen plasma units is still common treatment for iron deficiency anemia or reversal of oral anticoagulation. Awareness of such transfusion-associated risks as TRALI, should result in more restrictive indications for the use of blood in clinical practice.

\section{Conclusions}

TRALI is a serious, life-threatening complication related to the transfusion of blood components. After years of research, the pathogenesis of the syndrome is better understood, the diagnostic criteria have been established and preventive measures implemented. This contributed to the reduction of the overall number of fatalities. TRALI, however, still remains a clinical problem. Any complication suspected of TRALI should immediately be reported to the regional blood transfusion center, the diagnosis confirmed in laboratory tests and the look-back procedure launched (in Poland such diagnostics tests are performed at the Institute of Hematology and Transfusion Medicine in Warsaw). Correct diagnosis followed by proper management may save the lives of our patients as well as those who will require blood component transfusion in the future.

\section{Conflict of interest: none declared}

\section{References}

1. Barnard RD. Indiscriminate transfusion: a critique of case reports illustrating hypersensitivity reactions. NY State J Med, 1951; 51: 2399-2402.

2. Popovsky MA, Abel MD, Moore SB. Transfusion-related acute lung injury associated with passive transfer of antileukocyte antibodies. Am Rev Respir Dis, 1983; 128: 185-189.

3. Kleinman S, Caulfield T, Chan P et al. Toward an understanding of transfusion-related acute lung injury: statement of a consensus panel. Transfusion, 2004; 44: 1774-1789.

4. Swanson K, Dwyre DM, Krochmal J, Raife TJ. Transfusion-related acute lung injury (TRALI): current clinical and pathophysiologic considerations. Lung, 2006; 184: 177-185.

5. Müller MC, Juffermans NP. Transfusion-related acute lung injury: A preventable syndrome? Expert Rev Hematol, 2012; 5: 97-106.

6. Aravinthan A, Sen S, Marcus N. Transfusion-related acute lung injury: A rare and life-threatening complication of a common procedure. Clin Med, 2009; 9: 87-89.

7. Win N, Chapman CE, Bowles KM et al. How much residual plasma may cause TRALI? Transfus Med, 2008; 18: 276-280.

8. Looney MR, Gropper MA, Matthay MA. Transfusion-related acute lung injury: A review. Chest, 2004;126: 249-258.
9. http://www.fda.gov/BiologicsBloodVaccines/SafetyAvailability/ /ReportaProblem/TransfusionDonationFatalities/default.htm.

10. Shaz BH. Giving TRALI the one-two punch. Blood, 2012; 119: 1620-1621.

11. Sanchez R, Bacchetti P, Toy P. Transfusion-related acute lung injury: a case-control pilot study of risk factors. Am J Clin Pathol, 2007;128: 128-134.

12. Toy P, Popovsky MA, Abraham E et al. Transfusion-related acute lung injury: definition and review. Crit. Care Med, 2005; 33: 721-726.

13. Shaz BH, Stowell SR, Hillyer CD. Transfusion-related acute lung injury: From bedside to bench and back. Blood, 2011; 117: 1463-1471.

14. Bux J, Sachs UJ. The pathogenesis of transfusion-related acute lung injury (TRALI). Br J Haematol, 2007, 136: 788-799.

15. Maślanka K, Żupańska B. Immunological and non-immunological factors implicated in TRALI - Polish experience. J Transf Med, 2012; 5: 88-90.

16. Fung YL, Silliman CC. The role of neutrophils in the pathogenesis of transfusion-related acute lung injury. Transfus Med Rev, 2009; 23: 266-283.

17. Silliman CC, Moore EE, Kelher MR et al. Identification of lipids that accumulate during the routine storage of prestorage leukoreduced red blood cells and cause acute lung injury. Transfusion, 2011;51: 2549-2554.

18. Maślanka K, Smoleńska-Sym G, Michur H et al. Lysophosphatidylcholines: Bioactive lipids generated during storage of blood components. Arch Immunol Ther Exp, 2012; 60: 55-60.

19. Wróbel A, Maślanka K, Uhrynowska M et al. Microparticles in blood components transfused to patients with dyspnea-associated non-hemolytic transfusion reactions. JTransf Med, 2012; 5: 65.

20. Łopacz P, Wróbel A, Sak-Budzisz J, Lachert E, Maślanka K, Brojer E. Ocena stężenia IL-1beta, IL-6, IL-8, TNF-alpha i sCD40L w przechowywanych koncentratach erytrocytarnych i plytkowych. Acta Haematol Pol, 2011; 42: 85-93.

21. Virchis AE, Patel RK, Contreras M, Navarrete C, Kaczmarski RS, Jan-Mohamed R. Acute non-cardiogenic lung oedema after platelet transfusion. BMJ, 1997; 314: 880-822.

22. Eastlund DT, McGrath PC, Burkart P. Platelet transfusion reaction associated with interdonor HLA incompatibility. Vox Sang, 1988; 55: 157-160.

23. Dykes A, Smallwood D, Kotsimbos T, Street A. Transfusion-related acute lung injury (Trali) in a patient with a single lung transplant. Br J Haematol, 2000; 109: 674-676.

24. Maślanka K, Michur H, Zupańska B, Uhrynowska M, Nowak J. Leucocyte antibodies in blood donors and a look back on recipients of their blood components. Vox Sang, 2007; 92: 247-249.

25. Silliman CC, Ambruso DR, Boshkov LK. Transfusion-related acute lung injury. Blood, 2005; 105: 2266-2273.

26. Covin RB, Ambruso DR, England KM et al. Hypotension and acute pulmonary insufficiency following transfusion of autologous red blood cells during surgery: A case report and review of the literature. Transfus Med, 2004; 14: 375-383.

27. Zupanska B, Uhrynowska M, Michur H, Maślanka K, Zajko M. Transfusion-related acute lung injury and leucocyte-reacting antibodies. Vox Sang, 2007; 93: 70-77.

28. Vlaar AP, Hofstra JJ, Determann RM et al. The incidence, risk factors and outcome of transfusion-related acute lung injury in a cohort of cardiac surgery patients: a prospective nested case control study. Blood, 2011; 117: 4219-4225. 
29. Sachs UJ, Weissmann N, Wasel W et al. Supernatants from stored leukodepleted packed red blood cells do not regularly exhibit changes in their (lyso)phosphatidylcholine composition and do not cause TRALI in an ex vivo rat lung model. Vox Sang, 2010; 99 (Suppl. s1): 456.

30. Triulzi DJ. Transfusion-related acute lung injury: An update. Hematol Am Soc Hematol Educ Program, 2006: 497-501.

31. Bierling P, Bux J, Curtis B et al. Recommendations of the ISBT Working Party on Granulocyte Immunobiology for leucocyte antibody screening in the investigation and prevention of antibody-mediated transfusion-related acute lung injury. Vox Sang, 2009; 96: 266-269.

32. Reesink HW, Lee J, Keller A, Dennington P et al. Measures to prevent transfusion-related acute lung injury (TRALI). Vox Sang, 2012; 103: 231-259.

33. http://www.ihn-org.com/wp-content/uploads/2011/06/ISBT-definitions-for-non-infectious-transfusion-reactions.pdf.

34. Gajic O, Gropper MA, Hubmayr RD. Pulmonary edema after transfusion: How to differentiate transfusion-associated circulatory overload from transfusion-related acute lung injury. Crit Care Med, 2006; 34: 109-113.

35. Danielson C, Benjamin RJ, Mangano MM, Mills CJ, Waxman DA. Pulmonary pathology of rapidly fatal transfusion-related acute lung injury reveals minimal evidence of diffuse alveolar damage or alveolar granulocyte infiltration. Transfusion, 2008; 48: 2401-2408.

36. Roberts GH. Transfusion-related acute lung injury (TRALI). Clin Lab Sci, 2004; 17: 133-135.

37. Nouraei SM, Wallis JP, Bolton D, Hasan A. Management of transfusion-related acute lung injury with extracorporeal cardiopulmonary support in a four-year-old child. Br J Anaesth, 2003; 91: 292-294.

38. Wallis JP. Transfusion-related acute lung injury (TRALI)-under-diagnosed and under-reported. Br J Anaesth, 2003; 90: 573-576.

39. Lin Y, Kanani N, Naughton F, Pendergrast J, Karkouti K. Case report: Transfusion-related acute lung injury (TRALI) — A clear and present danger. Can J Anaesth, 2007; 54: 1011-1016.
40. Li G, Kojicic M, Reriani MK et al. Long-term survival and quality of life after transfusion-associated pulmonary edema in critically ill medical patients. Chest, 2010; 137: 783-789.

41. Kakaiya RM, Triulzi DJ, Wright DJ et al. Epidemiology Donor Study-II. Prevalence of HLA antibodies in remotely transfused or alloexposed volunteer blood donors. Transfusion, 2010; 50: 1328-1334.

42. Michala L, Madhavan B, Win N, De Lord C, Brown R. Transfusion-related acute lung injury (TRALI) in an obstetric patient. Int J Obstet Anesth, 2008; 17: 66-69.

43. Chapman CE, Stainsby D, Jones $\mathrm{H}$ et al. Ten years of hemovigilance reports of transfusion-related acute lung injury in the United Kingdom and the impact of preferential use of male donor plasma. Transfusion, 2009; 49: 440-452.

44. Lucas G, Win N, Calvert A et al. Reducing the incidence of TRALI in th UK: the results of screening for donor leucocyte antibodies and the development of national quidelines. Vox Sang, 2012; 102: 10-17.

45. Triulzi DJ, Kleinman S, Kakaiya RM et al. The effect of previous pregnancy and transfusion on HLA alloimmunization in blood donors: implications for a transfusion-related acute lung injury risk reduction strategy. Transfusion, 2009; 49: 1825-1835.

46. Kopko PM, Marshall CS, MacKenzie MR, Holland PV, Popovsky MA. Transfusion-related acute lung injury: report of a clinical look-back investigation. JAMA, 2002; 287: 1968-1971.

47. Lachert E, Antoniewicz-Papis J, Kubis J, Woźniak J, Łętowska M. PRT Mirasol System - validation experience in Poland. JTranf Med, 2012; 5: 91-93.

48. Krochmal JD, Dwyre DM, Swanson KM, Raife TJ, Schlueter AJ. Recurrent transfusion-related acute lung injury after a two-year interval. Transfus Med, 2007; 17: 192-196.

49. Win N, Montgomery J, Sage D, Street M, Duncan J, Lucas G. Recurrent transfusion-related acute lung injury. Transfusion, 2001; 41: 1421-1425.

50. Davis A, Mandal R, Johnson M, Makar R, Stowell C, Dzik S. A touch of TRALI. Transfusion, 2008; 48: 541-545. 